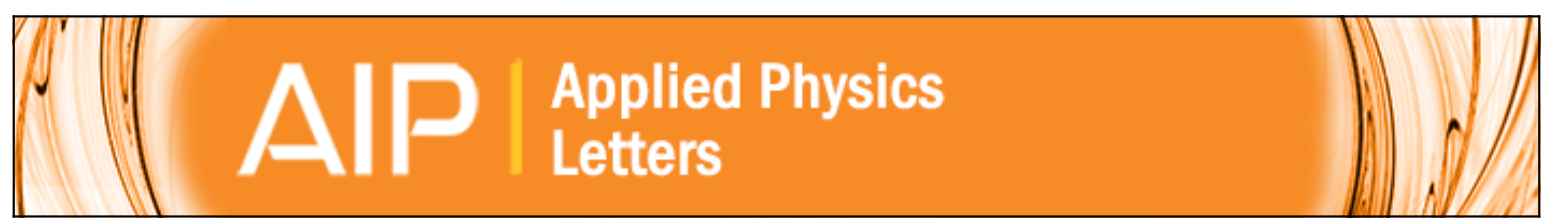

\title{
Terahertz generation in multiple laser-induced air plasmas
}

Meng-Ku Chen, Jae Hun Kim, Chia-En Yang, Stuart Shizhuo Yin, Rongqing Hui, and Paul Ruffin

Citation: Applied Physics Letters 93, 231102 (2008); doi: 10.1063/1.3046122

View online: http://dx.doi.org/10.1063/1.3046122

View Table of Contents: http://scitation.aip.org/content/aip/journal/apl/93/23?ver=pdfcov

Published by the AIP Publishing

\section{Articles you may be interested in}

The effect of blast wave re-focusing on a laser-induced plasma

J. Appl. Phys. 113, 103101 (2013); 10.1063/1.4794017

Coherent microwave radiation from a laser induced plasma

Appl. Phys. Lett. 101, 264105 (2012); 10.1063/1.4773240

Terahertz wave generation from gas plasma using a phase compensator with attosecond phase-control accuracy

Appl. Phys. Lett. 94, 021117 (2009); 10.1063/1.3068501

Enhancement of terahertz wave generation from laser induced plasma

Appl. Phys. Lett. 90, 141104 (2007); 10.1063/1.2719165

Terahertz emission profile from laser-induced air plasma

Appl. Phys. Lett. 88, 261103 (2006); 10.1063/1.2216025

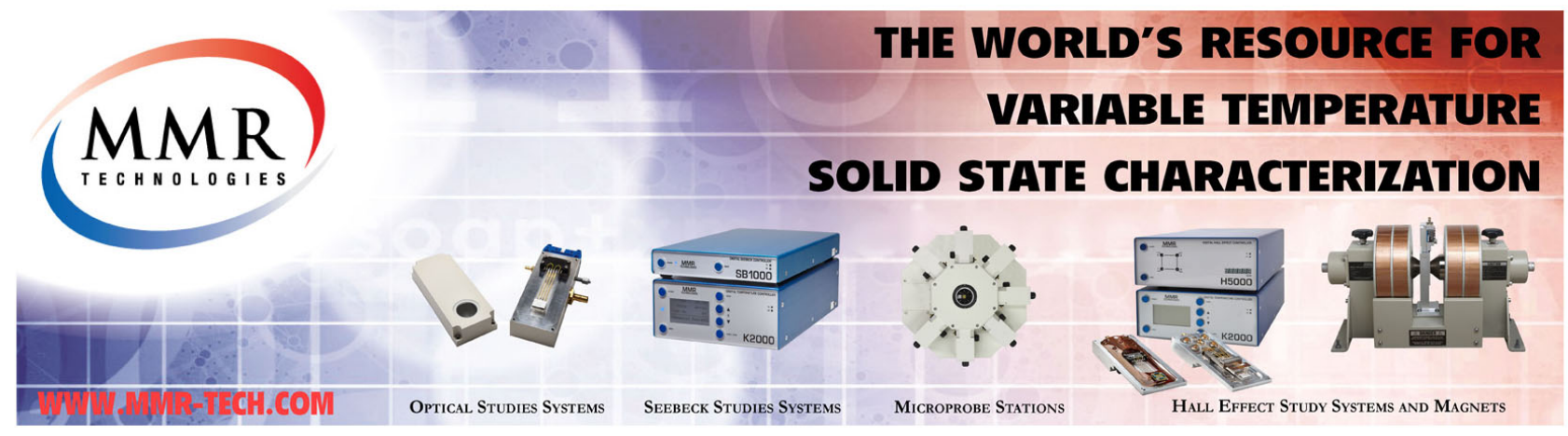




\title{
Terahertz generation in multiple laser-induced air plasmas
}

\author{
Meng-Ku Chen, ${ }^{1, a)}$ Jae Hun Kim, ${ }^{1}$ Chia-En Yang, ${ }^{1}$ Stuart Shizhuo Yin, ${ }^{1, b)}$ \\ Rongqing Hui, ${ }^{2}$ and Paul Ruffin ${ }^{3}$ \\ ${ }^{1}$ Department of Electrical Engineering, The Pennsylvania State University, University Park, \\ Pennsylvania 16802, USA \\ ${ }^{2}$ Department of Electrical Engineering and Computer Science, The University of Kansas, \\ Lawrence, Kansas 66045, USA \\ ${ }^{3}$ US Army Aviation and Missile Research Development and Engineering Center, Redstone Arsenal, \\ Alabama 35898, USA
}

(Received 10 October 2008; accepted 20 November 2008; published online 9 December 2008)

\begin{abstract}
An investigation of the terahertz wave generation in multiple laser-induced air plasmas is presented. First, it is demonstrated that the intensity of the terahertz wave increases as the number of air plasmas increases. Second, the physical mechanism of this enhancement effect of the terahertz generation is studied by quantitatively measuring the intensity of the generated terahertz wave as a function of phase difference between adjacent air plasmas. It is found out that the superposition is the main mechanism to cause this enhancement. Thus, the results obtained in this paper not only provide a technique to generate stronger terahertz wave but also enable a better understanding of the mechanism of the terahertz generation in air plasma. (C) 2008 American Institute of Physics. [DOI: $10.1063 / 1.3046122]$
\end{abstract}

Terahertz generation in air plasma has drawn a great deal of interest because it can produce a broadband terahertz signal $^{1}$ and has remotely controlled sensing capability, which greatly avoids the propagation loss because terahertz wave can be remotely (i.e., near the target location) generated and detected. Terahertz generation in laser induced air plasma was first observed by focusing high intensity single wavelength femtosecond laser pulses in air. ${ }^{2}$ Later, it was found that higher terahertz generation efficiency could be achieved by focusing and mixing the fundamental beam and its second harmonic beam together into air. ${ }^{3}$ Several physical mechanisms of terahertz generation by laser induced air plasma were also proposed, including (1) four-wave mixing, ${ }^{3}$ (2) ponderomotively induced space charge fields, ${ }^{2}$ and (3) the plasma current driven by an asymmetric laser field. ${ }^{4}$

Although the intensity of the terahertz wave has been substantially enhanced by employing the double wavelength technology (i.e., using both the fundamental and the second harmonic wave), the overall conversion efficiency is still very low (only a few percent or less). Most of the incoming laser light energy is not converted into the terahertz wave. Thus, there is a critical need to increase the conversion efficiency. Recent efforts of increasing terahertz generation efficiency included using preformed air plasma ${ }^{5}$ and terahertz wave amplification through four-wave-mixing parametric process. ${ }^{6}$ However, no more than two air plasmas have been used. Furthermore, there is also a lack of systematic study on the physical mechanism of the enhancement effect of the terahertz wave generation by using multiple air plasmas.

To further enhance terahertz wave generation efficiency and to have a better understanding of the physical mechanism of the enhancement effect of the terahertz wave generation by multiple air plasmas, in this paper, we report the enhanced terahertz wave generation by using more than two

\footnotetext{
${ }^{a)}$ Electronic mail: muc202@psu.edu.

b) Author to whom correspondence should be addressed. Electronic mail: sxy105@psu.edu.
}

(three) air plasmas. Furthermore, the physical mechanism of the enhancement effect of the terahertz wave generation by harnessing multiple air plasmas is also quantitatively studied by adjusting the phase difference between adjacent air plasmas. It confirms that superposition is the main mechanism for such kind of enhancement. Thus, the method reported in this paper not only provides a technique to enhance the terahertz wave generation efficiency but also offers a better understanding on the physical mechanism of terahertz wave generation when multiple air plasmas are involved.

The experimental setup is shown in Fig. 1. The laser beam from the regeneratively amplified Ti:sapphire laser system, which provides $\sim 150 \mathrm{fs}, 780 \mathrm{~nm}$ pulses at a repetition rate of $1 \mathrm{KHz}$, was split into two beams, which were the pump beam and the probe beam. The pump beam was first focused by the parabolic mirror 1 and then passed through a $100 \mu \mathrm{m}$ thick $\beta$-barium borate (BBO) crystal plate. The first air plasma was induced by the combination of the fundamental beam and its second harmonic beam, which enabled terahertz signal generation in the first air plasma. Then, all the beams were focused by a second parabolic mirror 2, which induced the second air plasma and generated second terahertz signal. Finally, a third air plasma, which was induced

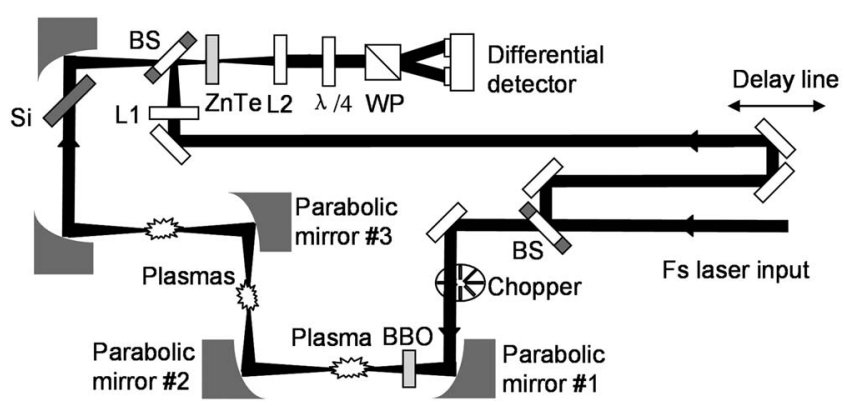

FIG. 1. Experimental setup of terahertz generation in multiple air plasmas. BS denotes beam splitter, L1 and L2 denotes lenses with focal lengths of 10 $\mathrm{cm}, \lambda / 4$ denotes the quarter-wave plate, and WP denotes Wollaston prism. 


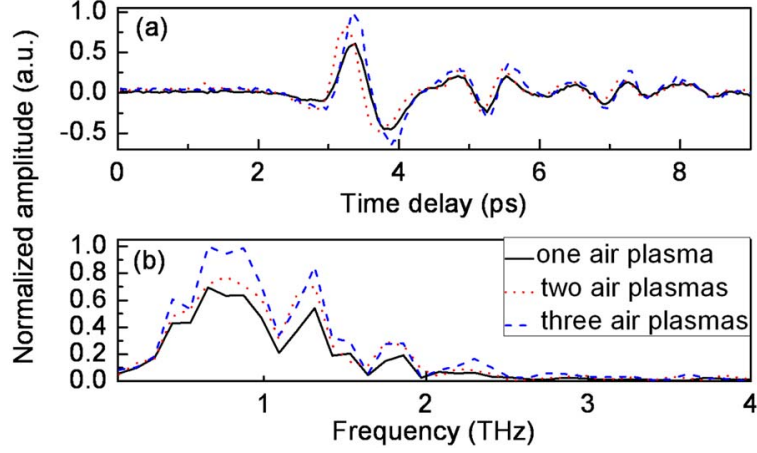

FIG. 2. (Color online) (a) Terahertz waveforms obtained by scanning probe beam delay line. (b) The corresponding spectra obtained from the Fourier transform of the terahertz waveforms.

by the beams focused by parabolic 3, generated the third terahertz signal. Note that in the experiment, all parabolic mirrors 1-3 are protected aluminum coating mirrors, which have good reflections at wavelength 390 and $780 \mathrm{~nm}$ and terahertz range. The silicon wafer was used to block all the beams except the terahertz beam. The terahertz beam and the probe beam are focused at the same spot of $1 \mathrm{~mm}\langle 110\rangle$ oriented ZnTe crystal. The electro-optic sampling method $^{7}$ was used for the terahertz wave detection. Both the terahertz beam and the probe beam were focused on a $\langle 110\rangle$ oriented ZnTe crystal with a spot size around $1 \mathrm{~mm}$.

Figure 2(a) shows the experimentally measured terahertz waveforms in the time domain obtained by the scanning probe beam delay line approach, and Fig. 2(b) shows the corresponding spectra obtained from the Fourier transform of the terahertz waveforms generated from one air plasma, two air plasmas, and three air plasmas, respectively. To investigate the relationship between the intensity of the generated terahertz signal and the number of the air plasmas, terahertz signal generated by one air plasma and two air plasmas were obtained by placing silicon wafer right after parabolic mirrors 2 and 3, respectively. In Fig. 2(a), we can observe that the peak terahertz amplitude obtained from two air plasmas is about $37 \%$ higher than that obtained from one air plasma, and the peak terahertz amplitude obtained from three air plasmas is about $20 \%$ higher than that obtained from two air plasmas. Similarly, the intensities of terahertz signal in spectral domain also increase as the number of the air plasma increases, as depicted in Fig. 2(b). Thus, one can conclude that the terahertz signal can indeed be enhanced by harnessing multiple air plasmas. Also, we believe that the terahertz signal is not doubled when using two air plasmas to replace one air plasma is mainly due to the partial absorption of the parabolic reflection mirrors and the slight phase mismatch between terahertz signals generated from different air plasmas, to be discussed in detail below.

One of the advantages of using parabolic mirrors is that we only need to consider the dispersion effect in air for the 780 and $390 \mathrm{~nm}$ beams. The refractive index of air at 780 and $390 \mathrm{~nm}$ is 1.000275 and $1.000283 .{ }^{8}$ The distance $d$ between the first and the third air plasmas is about $40 \mathrm{~cm}$. Therefore, the overall walk-off between 780 and $390 \mathrm{~nm}$ pulses can be calculated by $d\left(n_{390}-n_{780}\right)$ $=40 \mathrm{~cm} \times(1.000283-1.000275)=3.2 \mu \mathrm{m}$. Because the pulse length of the laser is about $45, \mu \mathrm{m}$, the two pulses

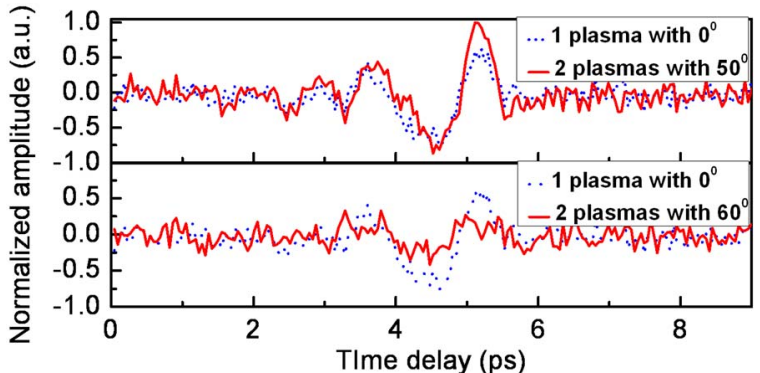

FIG. 3. (Color online) Terahertz waveforms obtained by scanning probe beam delay line with different incident angles on cover glass.

still overlap relatively well even with a $3.2 \mu \mathrm{m}$ walk-off distance.

To investigate the physical mechanism of the terahertz enhancement by employing multiple air plasmas, the following experiments were conducted. First, we extended investigations on the influence of the terahertz signals by the relative phase difference between the fundamental and the second harmonic exciting laser beams from one air plasma case $^{9}$ to the case of multiple air plasmas. In the experiment, this relative phase delay was realized by adjusting the distance between the BBO crystal and the first air plasma. We observed that the terahertz amplitude obtained from the first air plasma was changed, which was due to the phase shift between the fundamental and its second harmonic waves, as mentioned in Ref. 9. The new discoveries were that the enhancement ratios of terahertz wave generated for the cases of two air plasmas and three air plasmas were also changed, which suggested that terahertz amplitude generated in the second and the third air plasmas was also sensitive to the phase shift between the fundamental and second harmonic waves. To further verify this point, in the second experiment, we put a $110 \mu \mathrm{m}$ thick cover glass between the first and the second air plasmas. The terahertz waveform obtained from two air plasmas was changed when the cover glass was tilted, as shown in Fig. 3. With an incident angle of $50^{\circ}$, the upper curve as depicted in Fig. 3, the peak terahertz amplitude obtained from two air plasmas is about $60 \%$ higher than that obtained from one air plasma, which could be contributed to the constructive interference between the terahertz waves generated in the first air plasma and the second one. However, as for the case of the incident angle of $60^{\circ}$, the lower curve as depicted in Fig. 3, the deconstructive interference between the terahertz waves generated in the first air plasma and the second one may be responsible for the lower peak terahertz amplitude, which is even about $56 \%$ lower than that obtained from one air plasma. For the purpose of illustration, Fig. 4 shows the peak terahertz amplitude ob-

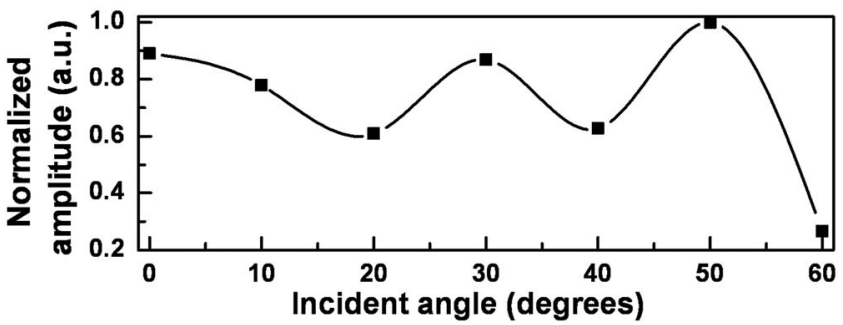

FIG. 4. The peak terahertz amplitude from two air plasmas as a function of incident angle on cover glass. 
tained from two air plasmas as a function of incident angle on cover glass. It clearly shows a periodic interference nature.

The above qualitatively description is also consistent with the mathematical models based on the four-wave mixing theory and the plasma current theory. The mathematical equations for the four-wave mixing theory ${ }^{9}$ and the plasma current model $^{1}$ are given by

$$
\begin{aligned}
& E_{\mathrm{THz}} \propto \chi^{(3)} E_{\omega}^{* 2} E_{2 \omega} \sin (\varphi), \\
& E_{\mathrm{THz}} \propto d J / d t \propto f\left(E_{\omega}\right) E_{2 \omega} \sin (\varphi),
\end{aligned}
$$

where $E_{\omega}$ and $E_{2 \omega}$ are the amplitudes of the fundamental and second harmonic waves, respectively, $J$ is the plasma current, and $\varphi$ is the relative phase between $\omega$ and $2 \omega$ waves. To adjust the phase difference between the fundamental and the second harmonic beams, a rotatable cover glass is used to introduce the adjustable phase change. Based on Eq. (1), the cover glass induced adjustable phase change $\Delta \varphi$ to the terahertz wave generation can be modeled by multiplying the phase terms of $E_{\omega}^{* 2}$ and $E_{2 \omega}$, as given by

$$
\Delta \varphi=\frac{2 \omega}{c} d\left(\frac{n_{\omega}}{\cos \alpha_{\omega}}-\frac{n_{2 \omega}}{\cos \alpha_{2 \omega}}\right),
$$

where $n_{\omega}$ and $n_{2 \omega}$ are the refractive indices of glass at the fundamental and the second harmonic frequencies, $d$ is the thickness of the cover glass, and $\alpha_{\omega, 2 \omega}=\sin ^{-1}\left(\sin \beta / n_{\omega, 2 \omega}\right)$, where $\beta$ is the incident angle on cover glass. This adjustable phase change $\Delta \varphi$ is continuously tuned by rotating the angle of cover glass so that a periodic change in the terahertz signal is observed, as illustrated in Fig. 4. Also, the experimental results obtained in this paper confirmed that the enhancement of total terahertz signals by using multiple air plasmas was due to the coherent superposition of individual terahertz signal from each air plasma because it was sensitive to the relative phase among different terahertz signals. Finally, we estimate the phase slippage between the fundamental and second harmonic waves in the air plasma, which can be expressed as $\Delta \theta=(3 \pi / 2)(L / \lambda)\left(N_{e} / N_{c}\right),{ }^{4}$ where $L \sim 5 \mathrm{~mm}$ is the length of air plasma, $\lambda=780 \mathrm{~nm}$ is the fundamental wavelength, $N_{c}=m_{e} \omega^{2} /\left(4 \pi e^{2}\right)=1.8 \times 10^{21} \mathrm{~cm}^{-3}$ is the critical density at $\lambda=780 \mathrm{~nm}$, and $N_{e}=1.2 \times 10^{16} \mathrm{~cm}^{-3}$ is the critical density obtained at $1 \mathrm{THz}$. The estimated phase slippage $\Delta \theta$ is about $0.2 \mathrm{rad}$, which means that the phasematching condition is still maintained well in the air plasma.

In summary, we demonstrated the enhancement of terahertz wave generation by employing multiple (more than two) laser-induced air plasmas. We also confirmed that the physical mechanism of this enhancement was due to the coherent superposition of the terahertz waves generated by each individual air plasma. The results reported in this paper will not only teach a technique on how to increase the efficiency of terahertz generation by harnessing multiple air plasmas but also provide a better understanding on the physical mechanism of the enhancement effect. This terahertz wave enhancement method provides an approach for the development of intense terahertz sources. Furthermore, terahertz generation in multiple air plasmas in cavity structure, which can induce many air plasmas by reflecting laser pulses back and forth inside the cavity, may be a possible way to further improve the enhancement of terahertz wave.

Partial financial support of this work by Office of Naval Research (Grant No. N00014-08-1-0538) is greatly appreciated. The authors also gratefully acknowledge helpful discussions and technical support from Zhiwen Liu and Peng Li.

${ }^{1}$ K. Y. Kim, A. J. Taylor, J. H. Glownia, and G. Rodriguez, Nat. Photonics 2, 605 (2008)

${ }^{2}$ H. Hamster, A. Sullivan, S. Gordon, and R. Falcone, Phys. Rev. E 49, 671 (1994).

${ }^{3}$ X. Xie, J. Dai, and X.-C. Zhang, Phys. Rev. Lett. 96, 075005 (2006).

${ }^{4}$ K. Y. Kim, J. H. Glownia, A. J. Taylor, and G. Rodriguez, Opt. Express 15, 4577 (2007).

${ }^{5}$ X. Xie, J. Xu, J. Dai, and X.-C. Zhang, Appl. Phys. Lett. 90, 141104 (2007).

${ }^{6}$ J. Dai, X. Xie, and X.-C. Zhang, Appl. Phys. Lett. 91, 211102 (2007)

${ }^{7}$ P. Planken, H.-K. Nienhuys, H. J. Bakker, and T. Wenckebach, J. Opt. Soc. Am. B 18, 313 (2001).

${ }^{8}$ P. Ciddor, Appl. Opt. 35, 1566 (1996).

${ }^{9}$ M. Kress, T. Löffler, S. Eden, M. Thomson, and H. Roskos, Opt. Lett. 29, 1120 (2004) 